Check for updates

Cite this: RSC Adv., 2019, 9, 27640

Received 9th July 2019

Accepted 28th August 2019

DOI: 10.1039/c9ra05201a

rsc.li/rsc-advances

\section{Polyacrylamide crosslinked by bis- vinylimidazolium bromide for high elastic and stable hydrogels}

\begin{abstract}
Caihong Wang, (D) Xiaoqin Guan, Yongli Yuan, Yong Wu (iD) and Shuai Tan (D)*
A series of ionic compounds $1, n$-dialkyl-3,3' -bis-l-vinylimidazolium bromide $\left(C_{n} V I M\right)$ are prepared and employed to crosslink acrylamide for polyacrylamide (PAAM) hydrogel preparation via in situ solution polymerization. The swelling behavior, mechanical properties and thermal stability of the prepared $\mathrm{C}_{n}$ VIM crosslinked PAAM hydrogels are investigated. $C_{n}$ VIM effectively crosslink the PAAM networks to form porous structures in the hydrogel, which could stably absorb water as much as 75.9 fold in weight without structural degradation. The prepared hydrogels could endure compressive stress up to $1.95 \mathrm{MPa}$ and compressive deformation more than $90 \%$. Meanwhile, the $C_{n}$ VIM crosslinked networks show superior thermal stability, and could retain the structural integrity under $150{ }^{\circ} \mathrm{C}$ for more than $240 \mathrm{~h}$. The swelling degradation resistance, mechanical strength and thermal stability of $\mathrm{C}_{n} \mathrm{VIM}$ crosslinked hydrogels are much better than those of a conventional $N, N^{\prime}$-methylenebisacrylamide crosslinked PAAM hydrogel. Using bis-vinylimidazolium bromides as crosslinkers provides an optional strategy for constructing thermally and mechanically robust hydrogel networks.
\end{abstract}

\section{Introduction}

Hydrogels, which are three-dimensional crosslinked hydrophilic networks absorbing large amounts of water, have attracted much attention due to their application in areas such as controlled drug delivery, tissue engineering, sensors, waste-water treatment and enhanced oil recovery. ${ }^{1-7}$ In most cases, the applications of the hydrogels require considerable toughness to withstand mechanical loads and deformations. Although specially engineered hydrogels are known to exhibit excellent physical properties, they may suffer from drastic strength deterioration under some harsh conditions..$^{7-9}$ For example, the inevitable swelling of the hydrogel under different osmotic pressure would partly break the crosslinking networks, which greatly weaken the mechanical toughness of the hydrogels and limit the applicability in biological applications. ${ }^{10}$ In the enhanced oil recovery fields, conventional polymer hydrogel systems are subject to fast thermal degradation for water management in high temperature petroleum reservoirs. ${ }^{\mathbf{1 1 , 1 2}}$ At present, developing tough hydrogels possessing sufficient mechanical strength under complex conditions remains a great challenge.

The crosslinking network plays a critical role for the stability of hydrogels, which leads researchers to seek suitable crosslinkers. Ionic liquids, which are defined as molten salts having melting points lower than $100{ }^{\circ} \mathrm{C}$, have proved quite versatile for enabling a range of exciting applications due to their unique properties such as nonvolatility, high thermal stability and

School of Chemical Engineering, Sichuan University, No. 24 South Section 1, Yihuan Road, Chengdu 610065, China.E-mail: tanshuai@scu.edu.cn friendly to environment. ${ }^{13}$ Ionic liquids with divinyl moieties could be employed as crosslinker to copolymerized with monomers for polymer preparation. ${ }^{\mathbf{1 4 - 1 6}}$ For example, Gordon used an ionic liquid 1,8-di(vinylimidazolium)-octane bis[(trifluoromethyl)sulfonyl] amide to copolymerize with an ionic liquid monomer. ${ }^{\mathbf{1 4}}$ The prepared poly(ionic liquids) was thermally robust at temperatures up to $250{ }^{\circ} \mathrm{C}$. Zhang et al. prepared superabsorbent polymers via copolymerization of acrylamide (AAM), acrylic acid and tetraallylammonium chloride. ${ }^{15}$ The resulted crosslinking networks were thermally stable and could still absorb a large amount of water at $250{ }^{\circ} \mathrm{C}$. In view of these, we envision that using ionic liquids as crosslinkers to construct hydrogel networks could improve the mechanical stability of the hydrogels to resist swelling and thermal degradation.

In this study, a series of ionic compounds $1, n$-dialkyl-3, 3 '-bisl-vinylimidazolium bromide $\left(\mathrm{C}_{n} \mathrm{VIM}, n=2,6,10\right)$ were prepared and utilized as crosslinkers for PAAM hydrogel preparation via in situ solution polymerization, as shown in Fig. 1. $\mathrm{C}_{n}$ VIM acted as effective crosslinkers in the copolymerization of hydrogels to form porous structures. Water swelling tests indicated that the $\mathrm{C}_{n}$ VIM crosslinked PAAM networks could stably absorb water up to $75.9 \mathrm{~g} \mathrm{~g}^{-1}$ to equilibrium. Compression tests suggested that $\mathrm{C}_{n}$ VIM crosslinked PAAM $\left(\mathrm{C}_{n}\right.$ VIM-PAAM) hydrogel showed good mechanical strength and toughness under compression, which could withstand stresses higher than $1 \mathrm{MPa}$ and compressive strains higher than $90 \%$ without fracture. Aging test revealed that $\mathrm{C}_{n}$ VIM-PAAM hydrogels possessed extraordinary thermal stability, which could tolerate $150{ }^{\circ} \mathrm{C}$ for more than $240 \mathrm{~h}$ with structural integrity. The swelling behavior, mechanical strength and thermal stability of $\mathrm{C}_{n}$ VIM-PAAM 


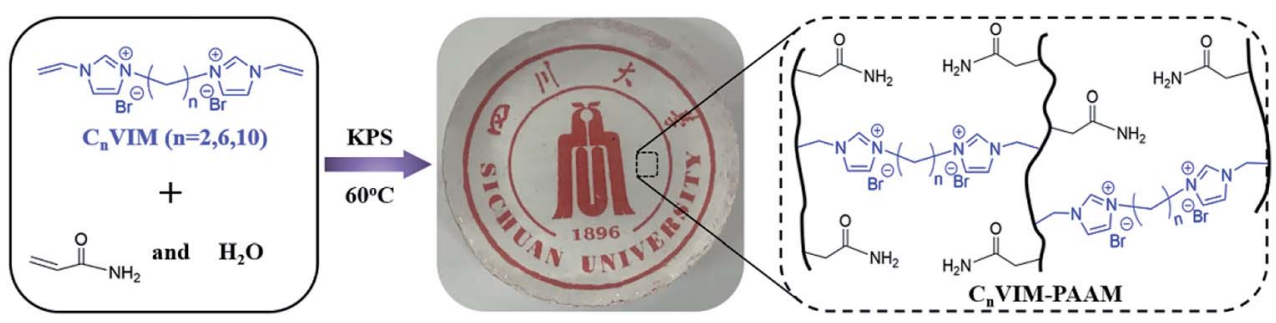

Fig. 1 Schematic diagram of $C_{n}$ VIM-PAAM hydrogel preparation and image of the resultant hydrogel.

hydrogel were much better than those of a $N, N^{\prime}$-methylenebisacrylamide (MBA) crosslinked PAAM hydrogel.

\section{Experimental}

\subsection{Materials}

All commercially-available starting materials, reagents and solvents were obtained from TCI and Acros and used as supplied.

\subsection{Characterization}

The ${ }^{1} \mathrm{H}$ NMR spectra were obtained in $\mathrm{D}_{2} \mathrm{O}$ using a Bruker AV II400 spectrometer. The Fourier transform IR spectra were measured with the use of a Perkin Elmer Spectrum Two Li10014 spectrometer. Before measurements, $\mathrm{C}_{n}$ VIM-PAAM were freezedried under vacuum to constant weight in a Christ Alpha 1-2LD vacuum freeze dryer to remove the water. The scanning electron microscope (SEM) observations of the freeze-dried hydrogel samples were performed by a Nova NanoSEM 450 field emission SEM at an accelerating voltage of $5 \mathrm{kV}$. The thermogravimetric (TG) analyses of the freeze-dried hydrogel samples were conducted on a TA Q600 gravimetric analyzer over a temperature range of $30-600{ }^{\circ} \mathrm{C}$ at a heating rate of $10{ }^{\circ} \mathrm{C} \mathrm{min}-1$ under nitrogen atmosphere. Dynamic mechanical analysis (DMA) was performed on a TA Q800 dynamic mechanical analyzer. The samples for DMA measurements were prepared in the form of cylinder ( $35 \mathrm{~mm}$ in diameter and $4 \mathrm{~mm}$ in height). The sample were first subjected to a strain sweep test at constant frequency $(1 \mathrm{~Hz})$ in which they were deformed at different compress strains, and the modulus Ge was recorded to define the linear viscoelastic region in which the modulus is independent of the strain. $1 \%$ deformation was chosen to ensure that each measurement was made in linear viscoelastic region in frequency sweep tests (from 0.1 to $10 \mathrm{~Hz}$ at $25{ }^{\circ} \mathrm{C}$ ). The compression tests were performed using an Instron 5967 electronic universal test machine at room temperatures. The compression tests of the samples $(10 \mathrm{~mm}$ in diameter and $10 \mathrm{~mm}$ in height) were performed with a crosshead speed of 5 $\mathrm{mm} \mathrm{min}^{-1}$. Compressive modulus was calculated from the slope of the linear region of the stress-strain curve during the compression tests (strain: 10-15\%). The fracture stress of the samples was defined as the stress at the breaking point.

\subsection{Synthesis of $1, n$-dialkyl-3,3'-bis-1-vinylimidazolium bromide ( $\left.\mathrm{C}_{n} \mathrm{VIM}\right)$}

All the bis-vinylimidazolium bromide crosslinkers were prepared by procedures analogous to that described below for
$\mathrm{C}_{6}$ VIM. 1,6-Dibromohexane (3 $\left.\mathrm{ml}, 10 \mathrm{mmol}\right)$ and 1-vinylimidazole $(2 \mathrm{ml}, 22 \mathrm{mmol})$ were dissolved in methanol $(5 \mathrm{~mL})$. The mixture was stirred at $60^{\circ} \mathrm{C}$ for $30 \mathrm{~h}$, then ethyl acetate $(50$ $\mathrm{ml}$ ) was added to precipitate resultant bis-vinylimidazolium bromide salt. The precipitate was separated through filtration, washed with ethyl acetate for twice and dried under vacuum to constant weight at $40{ }^{\circ} \mathrm{C}$ to give $\mathrm{C}_{6} \mathrm{VIM}(4.1 \mathrm{~g})$. Yield: $95 \% .{ }^{1} \mathrm{H}$ NMR (400 MHz, $\left.\mathrm{D}_{2} \mathrm{O}, \delta\right): 8.24,7.94(\mathrm{~d}, 4 \mathrm{H}, \mathrm{N}-\mathrm{CH}-\mathrm{CH}-\mathrm{N}), 7.36$ $\left(\mathrm{m}, 2 \mathrm{H}, \mathrm{CH}_{2}-\mathrm{CH}-\mathrm{N}\right), 5.69,5.31\left(\mathrm{~m}, 4 \mathrm{H}, \mathrm{CH}_{2}=\mathrm{CH}-\mathrm{N}\right), 4.17(\mathrm{t}$, $\left.4 \mathrm{H}, \mathrm{CH}_{2}-\mathrm{CH}_{2}-\mathrm{N}\right), 1.75$ (t, 4H, $\left.\mathrm{CH}_{2}-\mathrm{CH}_{2}-\mathrm{N}\right), 1.24$ (t, $4 \mathrm{H}, \mathrm{CH}_{2}-$ $\mathrm{CH}_{2}-\mathrm{CH}_{2}-\mathrm{N}$ ); IR (KBr): $\nu=3450,3137,3075,2931,2845,1646$, 1547, 1453, 1378, 1310, 1169, 1105, 957, 919, 830, 741, 630, $597 \mathrm{~cm}^{-1}$; anal. calcd for $\mathrm{C}_{16} \mathrm{H}_{24} \mathrm{Br}_{2} \mathrm{~N}_{4}$ : C 44.46, H 5.60, N 12.96; found: C 44.40, H 5.58, N 12.9 .

\subsection{Synthesis of PAAM hydrogels}

The $\mathrm{C}_{n}$ VIM-PAAM hydrogels were prepared by procedures analogous to that described below for $\mathrm{C}_{6}$ VIM-PAAM. The mole ratio of the crosslinker $\mathrm{C}_{n}$ VIM to the monomer AAM was controlled at $1: 220$. The composition of gelant solution for PAAM hydrogel preparation are listed in Table $1 . \mathrm{C}_{6} \mathrm{VIM}(0.28 \mathrm{~g}$, $0.64 \mathrm{mmol})$, AAM $(10 \mathrm{~g}, 140.8 \mathrm{mmol})$ and ammonium persulfate (APS, $0.04 \mathrm{~g}, 0.18 \mathrm{mmol}$ ) were dissolved in deionized water $(90$ $\mathrm{mL}$ ). The mixture was stirred at room temperature for $30 \mathrm{~min}$ for total dispersion. The gelant solution were sealed in a plastic mold and the polymerization of the gelant solution was carried out at $60^{\circ} \mathrm{C}$ for $6 \mathrm{~h}$. The resultant hydrogels were colorless and transparent, as shown in Fig. 1. IR (KBr): $\nu=3450,2935,1668$, $1458,1420,1343,1126,651 \mathrm{~cm}^{-1}$.

A MBA crosslinked PAAM hydrogel (MBA-PAAM) was also prepared as a reference by procedures analogous to $\mathrm{C}_{n} \mathrm{VIM}$ PAAM preparation. The mole ratio of BIS to acrylamide was also controlled at $1: 220$.

\section{Results and discussion}

\subsection{Hydrogel preparation}

The successful preparation of the ionic crosslinkers $\mathrm{C}_{n} \mathrm{VIM}$ and the $\mathrm{C}_{n}$ VIM-PAAM hydrogel were confirmed by ${ }^{1} \mathrm{H}$ NMR and IR spectra, as shown in Fig. 2. In the IR spectrum of AAM, the bands at $988 \mathrm{~cm}^{-1}$ and $962 \mathrm{~cm}^{-1}$ were ascribed to the bending vibration of the acryl bond. These bands disappeared in the spectrum of the freeze-dried $\mathrm{C}_{6}$ VIM-PAAM and a new band at $2935 \mathrm{~cm}^{-1}$ (the $\mathrm{C}-\mathrm{H}$ stretching vibration in PAAM main chains) appeared in the spectrum of the freeze-dried $\mathrm{C}_{6} \mathrm{VIM}$-PAAM, which suggested the successful polymerization of AAM. The 
Table 1 The composition of gelant solution for PAAM hydrogel preparation

\begin{tabular}{|c|c|c|c|c|c|c|c|}
\hline Hydrogel & $\mathrm{C}_{2} \mathrm{VIM}(\mathrm{g})$ & $\mathrm{C}_{6} \mathrm{VIM}(\mathrm{g})$ & $\mathrm{C}_{10}$ VIM $(\mathrm{g})$ & MBA (g) & AAM $(g)$ & APS $(g)$ & $\mathrm{H}_{2} \mathrm{O}(\mathrm{g})$ \\
\hline $\mathrm{C}_{2}$ VIM-PAAM & 0.24 & - & - & - & 10 & 0.04 & 90 \\
\hline $\mathrm{C}_{6}$ VIM-PAAM & - & 0.28 & - & - & 10 & 0.04 & 90 \\
\hline MBA-PAAM & - & - & - & 0.1 & 10 & 0.04 & 90 \\
\hline
\end{tabular}

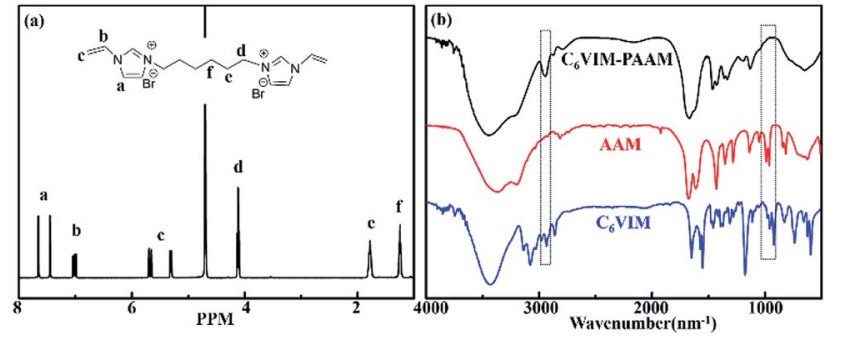

Fig. 2 (a) ${ }^{1} \mathrm{H}$ NMR spectrum of $\mathrm{C}_{6} \mathrm{VIM}$ in $\mathrm{D}_{2} \mathrm{O}$; (b) IR spectra of $\mathrm{C}_{6} \mathrm{VIM}$, AAM and dried $\mathrm{C}_{6}$ VIM-PAAM.

bands at $3450 \mathrm{~cm}^{-1}, 1668 \mathrm{~cm}^{-1}, 1458 \mathrm{~cm}^{-1}, 1420 \mathrm{~cm}^{-1}$, $1343 \mathrm{~cm}^{-1}, 1126$ and $651 \mathrm{~cm}^{-1}$ in the FT-IR spectrum of the freeze-dried $\mathrm{C}_{6}$ VIM-PAAM were ascribed to the $\mathrm{N}-\mathrm{H}$ stretching vibration, the $\mathrm{C}-\mathrm{H}$ stretching vibration in the $\mathrm{C}=\mathrm{O}$ stretching vibration of the amide group, the $\mathrm{CH}_{2}$ scissoring vibration, the C-N stretching vibration of the amide group, the $\mathrm{NH}_{2}$ scissoring vibration, the $\mathrm{N}-\mathrm{H}$ in-plane vibration and the $\mathrm{N}-\mathrm{H}$ out-of-plane vibration, respectively. ${ }^{17}$ The FT-IR spectra of freeze-dried $\mathrm{C}_{n}$ VIM-PAAM were substantially identical to that of freezedried $\mathrm{C}_{6}$ VIM-PAAM.

The microstructures of the prepared $\mathrm{C}_{n}$ VIM-PAAM hydrogels were characterized by SEM analysis. The SEM images of freezedried $\mathrm{C}_{n}$ VIM-PAAM hydrogels were shown in Fig. 3. Honeycomb-like structure formed in the $\mathrm{C}_{n}$ VIM-PAAM hydrogels, indicating the successful formation of crosslinking networks. The average pore size of $\mathrm{C}_{n}$ VIM-PAAM was about 10 $\mu \mathrm{m}$.

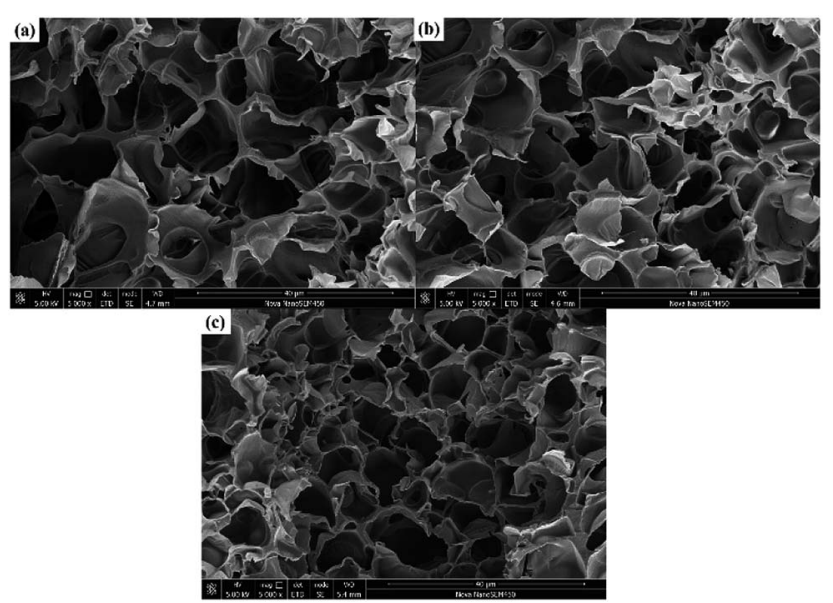

Fig. 3 SEM images of (a) freeze-dried $C_{2}$ VIM-PAAM, (b) $C_{6}$ VIMPAAM, (c) $\mathrm{C}_{10}$ VIM-PAAM.

\subsection{Swelling behavior}

The water swelling behavior of the hydrogels was determined using a gravimetric method at room temperature. Before measurements, all the as-prepared hydrogels were shaped in same size. The samples were immersed into the deionized water and weighted at specific time until they reached swelling equilibrium to determine the water swelling ratio. Afterwards, the hydrogels were dried in a vacuum oven at $60{ }^{\circ} \mathrm{C}$ to constant weight. The water swelling ratio (SR) and equilibrium water content (EWC) of the hydrogels were calculated using the following equations:

$$
\begin{gathered}
\mathrm{SR}=\frac{W_{\mathrm{s}}}{W_{\mathrm{o}}} \\
\mathrm{EWC}=\frac{W_{\mathrm{sE}}-W_{\mathrm{d}}}{W_{\mathrm{d}}}
\end{gathered}
$$

where $W_{\mathrm{o}}, W_{\mathrm{s}}, W_{\mathrm{sE}}$ and $W_{\mathrm{d}}$ stand for the weights of the asprepared hydrogel, the swollen hydrogel, the equilibrium swollen hydrogel and the totally dried hydrogel. The obtained swelling behavior of the hydrogels are shown in Fig. 4.

As shown in Fig. 4 a, the swelling ratio of the $\mathrm{C}_{n}$ VIM-PAAM hydrogels grew rapidly in the initial $40 \mathrm{~h}$ and reached a steady state after about $120 \mathrm{~h}$. The swelling ratio and the calculated equilibrium water content (inset of Fig. 4a) of $\mathrm{C}_{n}$ VIM-PAAM hydrogels increased with the length of the flexible alkyl chain in $\mathrm{C}_{n}$ VIM. The $\mathrm{C}_{n}$ VIM with longer flexible alkyl chain might form more loose crosslinking network to absorb more water. The equilibrium water content of the $\mathrm{C}_{10}$ VIM crosslinked PAAM networks could reached up to $75.9 \mathrm{~g} \mathrm{~g}^{-1}$. The $\mathrm{C}_{n}$ VIM crosslinked PAAM networks were robust to resist water swelling degradation. In spite of a high water content after equilibrium swelling, the equilibrium $\mathrm{C}_{n}$ VIM-PAAM hydrogels almost retained its

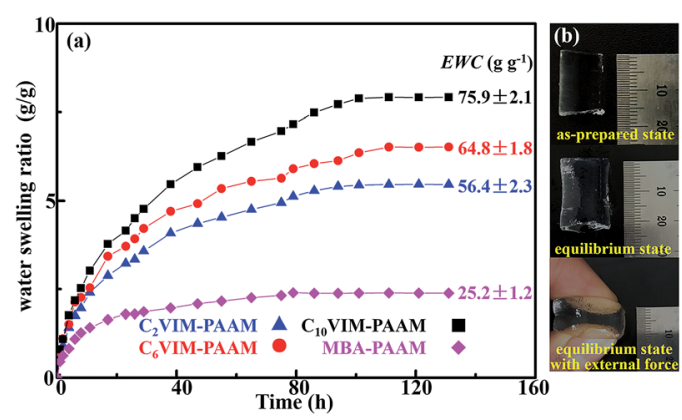

Fig. 4 (a) SR and EWC of the hydrogels as a function of time; (b) images of $\mathrm{C}_{6}$ VIM-PAAM hydrogel under as-prepared state and equilibrium state. 
original volume and shape, and could still withstand external force (Fig. 4b). This result indicated "noneswellable" properties of $\mathrm{C}_{n}$ VIM-PAAM hydrogels, which was important for hydrogel application..$^{\mathbf{8} 18}$ The $\mathrm{C}_{n}$ VIM-PAAM hydrogels swelled water faster and exhibited higher swelling ratio than the MBA-PAAM hydrogel, probably due to the strong hydrophilicity of the imidazolium moieties in the hydrogel networks.

\subsection{Mechanical property}

The viscoelastic properties of the $\mathrm{C}_{n} \mathrm{VIM}$-PAAM hydrogels were revealed by DMA tests. The measured elastic modulus of the asprepared hydrogels as a function of frequency are shown in Fig. 5. For all the hydrogels, the storage modulus was always much higher than the loss modulus over the entire frequency range, which indicated that $\mathrm{C}_{n}$ VIM-PAAM formed a stable three-dimensional network. ${ }^{19}$ The storage modulus of $\mathrm{C}_{n} \mathrm{VIM}-$ PAAM increased slightly with the increase in shear frequency, which was a consequence of the viscoelastic nature of the hydrogel. ${ }^{20}$ The flexible alkyl spacer in the crosslinker would reduce the rigidity of the crosslinking network in the hydrogels. As a result, the storage modulus of $\mathrm{C}_{n}$ VIM-PAAM decreased with the elongation of the alkyl chain in the crosslinker. All the $\mathrm{C}_{n}$ VIM-PAAM hydrogels exhibited plateau storage modulus higher than $30 \mathrm{kPa}\left(35.0 \pm 0.6 \mathrm{kPa}\right.$ for $\mathrm{C}_{2}$ VIM-PAAM, $33.8 \pm 0.4$ $\mathrm{kPa}$ for $\mathrm{C}_{6}$ VIM-PAAM and $30.8 \pm 0.7 \mathrm{kPa}$ for $\mathrm{C}_{10} \mathrm{VIM}-\mathrm{PAAM}$ ), which was higher than previously reported PAAM hydrogels. ${ }^{21-23}$ This result indicated the good viscoelastic property of the $\mathrm{C}_{n} \mathrm{VIM}$ crosslinked networks. The storage modulus of $\mathrm{C}_{n} \mathrm{VIM}-$ PAAM was also slightly higher than that of MBA-PAAM hydrogel, which might be ascribed to the rigid imidazolium moieties in the crosslinked networks.

The mechanical properties of the $\mathrm{C}_{n}$ VIM-PAAM hydrogels were revealed by uniaxial compressive tests. Compressive stress-strain curves of the hydrogels at a crosshead speed of 5 $\mathrm{mm} \min ^{-1}$ are shown in Fig. 6a. For $\mathrm{C}_{n} \mathrm{VIM}-\mathrm{PAAM}$, the compressive stress gradually increased with the increase in strain till the strain reached about $70 \%$. After that, the stress suddenly increased sharply. All the $\mathrm{C}_{n}$ VIM-PAAM hydrogels could withstand a compressive deformation of $90 \%$ strain and a compress stress of $1 \mathrm{MPa}$ without facture, which revealed good mechanical strength and toughness of the hydrogels. The compressive modulus, the fracture stress and the fracture strain of the hydrogels obtained from the curves are listed in Fig. 6a.

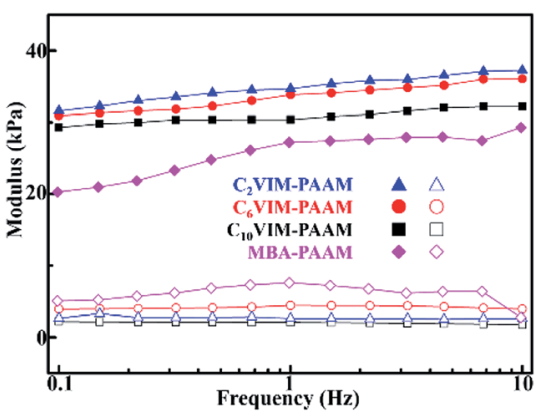

Fig. 5 The storage modulus (solid symbols) and loss modulus (open symbols) of the hydrogels at $25^{\circ} \mathrm{C}$ obtained from DMA tests.

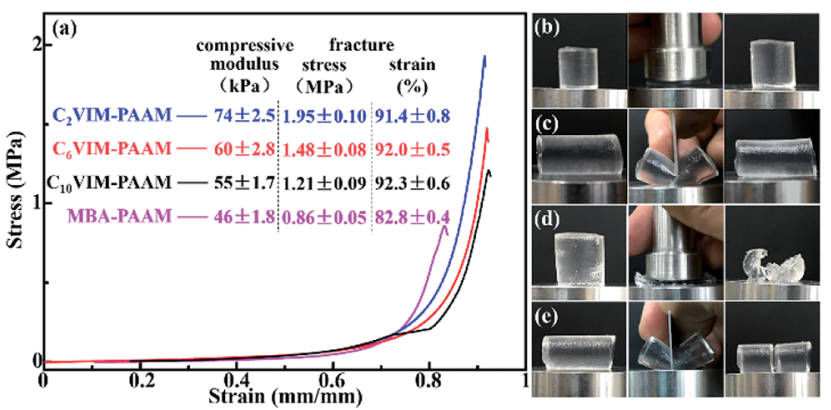

Fig. 6 (a) Compressive stress-strain curves of the hydrogels (inset is the compressive modulus, the fracture stress and the fracture strain of the hydrogels obtained from the curves); (b) and (c) images of the $\mathrm{C}_{6}$ VIM-PAAM hydrogel under compression and slicing with a blade, respectively; (d) and (e) images of the MBA-PAAM hydrogel under compression and slicing with a blade, respectively.

With the increasing in the length of the crosslinkers in $\mathrm{C}_{n} \mathrm{VIM}-$ PAAM hydrogels, the compressive modulus and the fracture stress of $\mathrm{C}_{n}$ VIM-PAAM hydrogels decreased, but the fracture strain increased. The $\mathrm{C}_{n}$ VIM-PAAM hydrogel crosslinked by shorter crosslinker might formed more rigid network with a higher deformation resistance, which needed more stress to achieve compressive deformation. Correspondingly, the $\mathrm{C}_{n}$ VIM-PAAM hydrogel crosslinked by longer crosslinker might formed more flexible network to allow larger deformation. $\mathrm{C}_{n}$ VIM-PAAM hydrogels exhibited a better performance to withstand compressive stress and deformation compared with MBA-PAAM hydrogel, which indicated a more efficient energy dissipation in $\mathrm{C}_{n}$ VIM-PAAM. The mechanical strength of $\mathrm{C}_{n}$ VIM-PAAM hydrogels was also higher than that of the reported chemically crosslinked hydrogels. ${ }^{24,25} \mathrm{C}_{n}$ VIM-PAAM hydrogels also exhibited better performance to sustain slicing than MBA-PAAM hydrogel (Fig. 6c and e), and showed good and fast recoverability, which could recover its original shape immediately after the external force released (Fig. $6 \mathrm{~b}$ and c).

\subsection{Thermal stability}

The thermal stability of the $\mathrm{C}_{n}$ VIM crosslinked networks was firstly characterized by TG analysis. All the freeze-dried $\mathrm{C}_{n} \mathrm{VIM}-$ PAAM hydrogels exhibited a similar decomposition process. Take $\mathrm{C}_{6}$ VIM-PAAM as an example (shown in Fig. 7), the weight loss below $120^{\circ} \mathrm{C}$ was ascribed to the dehydration. After dehydration, the weight loss followed a degradation mechanism with two major steps. The onset decomposition temperature of $\mathrm{C}_{6} \mathrm{VIM}-$ PAAM was $263^{\circ} \mathrm{C}$. The first decomposition step of $\mathrm{C}_{6}$ VIM-PAAM occurred from 263 to $345{ }^{\circ} \mathrm{C}$ arose likely from the elimination of ammonia gas from amide groups of PAAM chains. The second decomposition step occurred from 330 to $500{ }^{\circ} \mathrm{C}$, which was attributed to the decomposition of the polymer backbone and the cross-linked network structure. ${ }^{26}$ MBA-PAAM hydrogels also decomposed in two steps similarly to $\mathrm{C}_{6}$ VIM-PAAM, but showed a relatively poor thermal stability of the polymer backbone than $\mathrm{C}_{n}$ VIM-PAAM hydrogels, as shown in Fig. 7.

The as-prepared hydrogels $(35 \mathrm{~mm}$ in diameter and $40 \mathrm{~mm}$ in height) were further sealed in a stainless mold to perform 


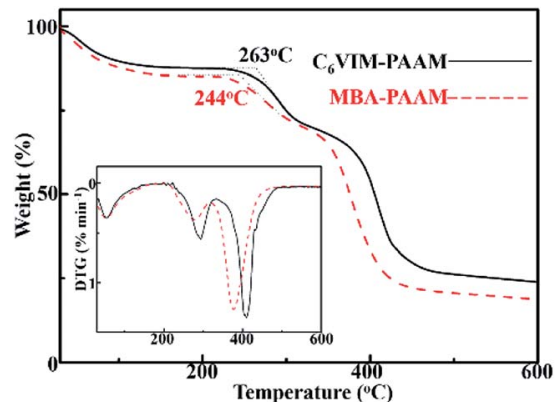

Fig. 7 TGA curves of the freeze-dried $\mathrm{C}_{6}$ VIM-PAAM and MBA-PAAM hydrogels (inset is the DTG curves of the hydrogels).

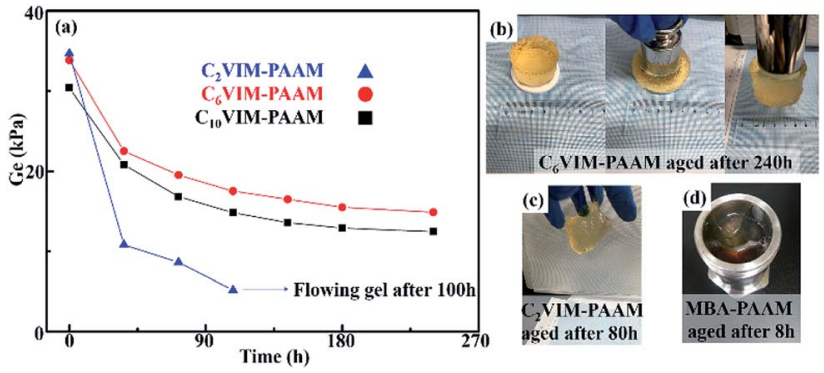

Fig. 8 (a) Plateau storage modulus of $C_{n}$ VIM-PAAM hydrogels aged at $150{ }^{\circ} \mathrm{C}$ as a function of time; (b) images of $\mathrm{C}_{6}$ VIM-PAAM hydrogel aged at $150{ }^{\circ} \mathrm{C}$ for $240 \mathrm{~h}$; (c) image of $\mathrm{C}_{2} \mathrm{VIM}$-PAAM hydrogel aged at $150^{\circ} \mathrm{C}$ for $80 \mathrm{~h}$; (d) images of MBA-PAAM hydrogels aged at $150{ }^{\circ} \mathrm{C}$ for $8 \mathrm{~h}$.

aging test at $150{ }^{\circ} \mathrm{C}$ and the thermal stability of the hydrogel were determined by the remained storage modulus of the aged hydrogels, as shown in Fig. 8a. For all the $\mathrm{C}_{n}$ VIM-PAAM hydrogels, the modulus decreased sharply in the initial $80 \mathrm{~h}$ and the colorless hydrogels became slight yellow (Fig. 8b and c). $\mathrm{C}_{6}$ VIM-PAAM and $\mathrm{C}_{10}$ VIM-PAAM hydrogels showed excellent thermal stability during the aging tests. After being aged at $150{ }^{\circ} \mathrm{C}$ for $240 \mathrm{~h}$, both the hydrogels could retain more than $40 \%$ the original modulus. The aged hydrogels remained stable in shape and could still withstand external force and compressive strain, as shown in Fig. 8b. However, $\mathrm{C}_{2}$ VIM-PAAM hydrogel consumed more than $80 \%$ the initial modulus, which lost its initial shape and became a flowing gel unable for further DMA tests after being aged for $100 \mathrm{~h}$, as shown in Fig. 8c. Despite the relatively poor stability compared with $\mathrm{C}_{6}$ VIM-PAAM and $\mathrm{C}_{10}$ VIM-PAAM hydrogels, the thermal stability of $\mathrm{C}_{2}$ VIM-PAAM hydrogel was still much superior than that of MBA-PAAM hydrogel. MBA-PAAM hydrogel lost the hydrogel nature and became a liquid within $8 \mathrm{~h}$ after being aged at $150{ }^{\circ} \mathrm{C}$, as shown in Fig. 8d. These results suggested that using $\mathrm{C}_{n} \mathrm{VIM}$ as crosslinkers for PAAM hydrogel preparation greatly improved the thermal stability of the crosslinked networks.

\section{Conclusions}

A series of polyacrylamide hydrogel were prepared via in situ solution copolymerization using bis-vinylimidazolium bromides as crosslinkers. The bis-vinylimidazolium bromides crosslinked networks showed good swelling degradation resistance with equilibrium water contents up to $75.9 \mathrm{~g} \mathrm{~g}^{-1}$. The prepared hydrogels were mechanically strong under compression with a compress modulus higher than $55 \mathrm{kPa}$ and a fracture stress higher than 1.2 $\mathrm{MPa}$ and a fracture deformation higher than $90 \%$ strain. More importantly, the hydrogels showed superior thermal stability, which could tolerate $150{ }^{\circ} \mathrm{C}$ for more than $240 \mathrm{~h}$ without fracture. Our work here shows the great potential of the bis-vinylimidazolium bromides as optional crosslinkers for PAAM hydrogels towards real world applications, such as water management in high-temperature petroleum reservoirs.

\section{Conflicts of interest}

There are no conflicts to declare.

\section{Acknowledgements}

This work was supported by the National Natural Science Foundation of China (No. 21805199).

\section{Notes and references}

1 K. Lee and D. Mooney, Chem. Rev., 2001, 101, 1869.

2 Y. Qiu and K. Park, Adv. Drug Delivery Rev., 2001, 53, 321.

3 M. Baker, S. Walsh, Z. Schwartz and B. Boyan, J. Biomed. Mater. Res., Part B, 2012, 100, 1451.

4 J. Holtz and S. Asher, Nature, 1997, 389, 829.

5 S. Basak, N. Nandi, S. Paul, I. W. Hamley and A. Banerjee, Chem. Commun., 2017, 53, 5910.

6 N. Nandi, K. Gayen, S. Ghosh, D. Bhunia, S. Kirkham, S. K. Sen, S. Ghosh, I. W. Hamley and A. Banerjee, Biomacromolecules, 2017, 18, 3621.

7 D. Zhu, B. Bai and J. Hou, Energy Fuels, 2017, 31, 13063.

8 H. Kamata, Y. Akagi, Y. Kayasuga-Kariya, U. Chung and T. Sakai, Science, 2014, 343, 873.

9 E. Appel, J. Barrio, X. Loh and O. Scherman, Chem. Soc. Rev., 2012, 41, 6195.

10 T. Nakajima, T. Kurokawa, S. Ahmed, W. Wu and J. Gong, Soft Matter, 2013, 9, 1955.

11 M. Caulfield, G. Qiao and D. Solomon, Chem. Rev., 2002, 102, 3067.

12 D. Zhu, J. Hou, X. Meng, Z. Zheng, Q. Wei, Y. Chen and B. Bai, Energy Fuels, 2017, 31, 8120.

13 S. Zhang, J. Zhang, Y. Zhang and Y. Deng, Chem. Rev., 2017, 117, 6755.

14 M. Muldoon and C. Gordan, J. Polym. Sci., Part A: Polym. Chem., 2004, 42, 3865.

15 X. Zhang, X. Wang, L. Li, S. Zhang and R. Wu, React. Funct. Polym., 2015, 87, 15.

16 P. Kasák, J. Mosnáček, M. Danko, I. Krupa, G. Hloušková, D. Chorvát, M. Koukaki, S. Karamanou, A. Economou and I. Lacík, RSC Adv., 2016, 6, 83890.

17 L. Chiem, L. Huynh, J. Ralston and D. Beattie, J. Colloid Interface Sci., 2006, 297, 54. 
18 N. Yuan, L. Xu, L. Zhang, H. Ye, J. Zhao, Z. Liu and J. Rong, Mater. Sci. Eng., C, 2016, 67, 221.

19 M. Song, Y. Wang, B. Wang, X. Liang, Z. Chang, B. Li and S. Zhang, ACS Appl. Mater. Interfaces, 2018, 10, 15021.

20 G. Song, Z. Zhao, X. Peng, C. He, R. Weiss and H. Wang, Macromolecules, 2016, 49, 8265-8273.

21 Y. Ohsedo, M. Taniguchi, K. Saruhashi and H. Watanabe, RSC Adv. , 2015, 5, 90010.

22 F. Selen, V. Can and G. Temel, RSC Adv., 2016, 6, 31692.
23 P. Tongwa, R. Nygaard and B. Bai, J. Appl. Polym. Sci., 2013, 38258.

24 M. Hu, X. Gu, Y. Hu, T. Wang, J. Huang and C. Wang, Macromolecules, 2016, 49, 3174.

25 M. Song, Y. Wang, B. Wang, X. Liang, Z. Chang, B. Li and S. Zhang, ACS Appl. Mater. Interfaces, 2018, 10, 15021.

26 R. Zolfaghari, A. Katbab, J. Nabavizadeh, R. Tabasi and M. Nejad, J. Appl. Polym. Sci., 2006, 100, 2096. 